\title{
Assembly of Nanoparticles into "Colloidal Molecules": Toward Complex and yet Defined Colloids with Exciting Perspectives
}

\author{
Claudia Simone Plüisch and Alexander Wittemann
}

Additional information is available at the end of the chapter

http://dx.doi.org/10.5772/65343

\begin{abstract}
In line with atoms being the elementary units of molecules and crystals, colloidal particles can be used as building blocks for organized materials. A major benefit in doing so is that joining colloids in a defined manner comes along with structuring. In view of opening avenues to more complex structural motifs, significant efforts must be geared to colloids with specific shapes and symmetries. A straightforward strategy is joining equal-sized spherical particles into stable clusters. Such clusters are called "colloidal molecules" because they may exhibit configurations resembling pretty much those of molecules. Their preparation can be based on the agglomeration of particles dispersed in an emulsion. The particles adsorb on the emulsion droplets and coagulate in a defined way during the evaporation of the droplet phase. Using this method originally applied to microscale particles, one can produce clusters with submicronsized global dimensions. Variable parameters such as radii and concentration of cluster constituents provide the framework needed to obtain "colloidal molecules" that differ in size, shape, and physical properties. This opens up exciting perspectives for tailormade colloids as building units for hierarchically organized materials. Moreover, new physical properties such as plasmonic "hotspots" may emerge from packing particles into assemblies of specific configurations.
\end{abstract}

Keywords: supracolloids, assemblies, nanoparticle clusters, heteroaggregates, colloidosomes, emulsion droplets, plasmonic colloids, depolarized dynamic light scattering, surface-enhanced Raman scattering 


\section{Introduction}

The preparation of suitable building blocks is the first step for the creation of new hierarchically organized materials, which is comparable to the construction of a building from single bricks. The elementary units and their interactions not only determine decisive properties of the material, but also regulate its genesis. In this context, the shape of the building blocks is particularly important [1], as it might be used to manipulate particle organization into desired hierarchically organized superstructures and, ultimately, materials (Figure 1).

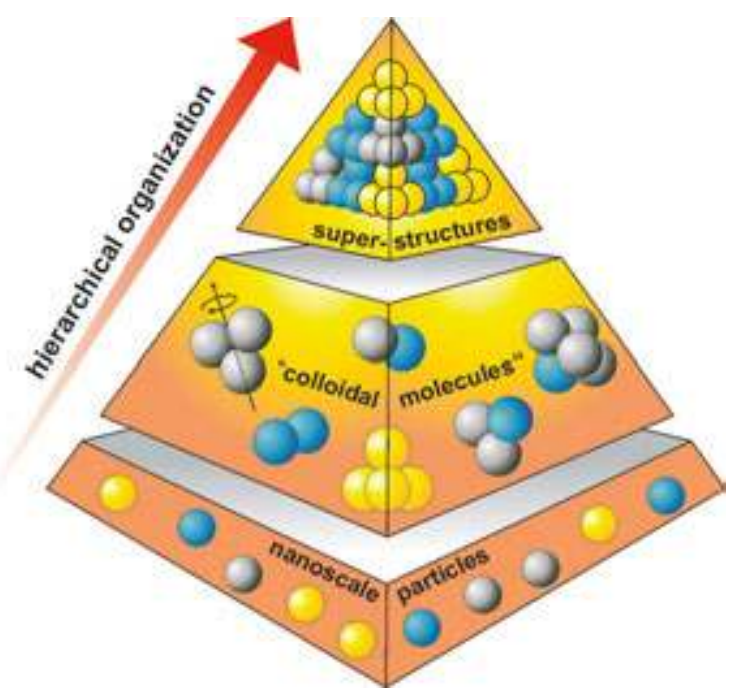

Figure 1. Perspectives of "colloidal molecules": Clusters of spherical elementary units have the potential to bridge the gap between single particles at the nanoscale and hierarchically organized materials at the macroscale. Moreover, they can serve as models for particles with complex shapes, or offer unique properties that emerge from packing nanoparticles into supraparticles.

However, minimization of surface tension favors the formation of spherical or quasi-spherical particle shapes. Under specific reaction conditions, which allow kinetically controlled growth, particle formation can be directed in an anisotropic dimension. Gold nanorods are probably the most prominent example of anisotropic inorganic colloids since the establishment of a wetchemistry approach for their synthesis [2]. With regard to polymer colloids, dumbbell-shaped particles can be prepared by controlled phase separation during seeded polymerization (Figure 2A). These particles can be used again as seeds for the preparation of trimers or even tetramer particles [3]. Dumbbell-shaped particles were successfully used as elementary units to build dense colloidal crystals with a partial band gap and birefringence [4]. Nucleation and growth of polymer nodules on spherical seeds give access to multipod-like or raspberry-like colloids [5]. Because of the organized coordination of the polymer nodules with regard to the seed, such complex particles are often called "colloidal molecules," if their subunits are seen as their "colloidal atoms" [6, 7]. In this terminology, the number of polymer nodules per central 
sphere equals the number of "free valences" that if made "sticky" could act as docking sites to build up hierarchically organized superstructures [8]. Spherical "Janus particles", i.e., particles with hemispherical coatings, can be seen as the simplest representatives of "colloidal molecules."Janus colloids already have shown a rich variety of superstructures [9]. "Colloidal molecules" of higher complexity are often considered to be model systems for molecular matters but also to function as building units for new hierarchically organized materials [10].

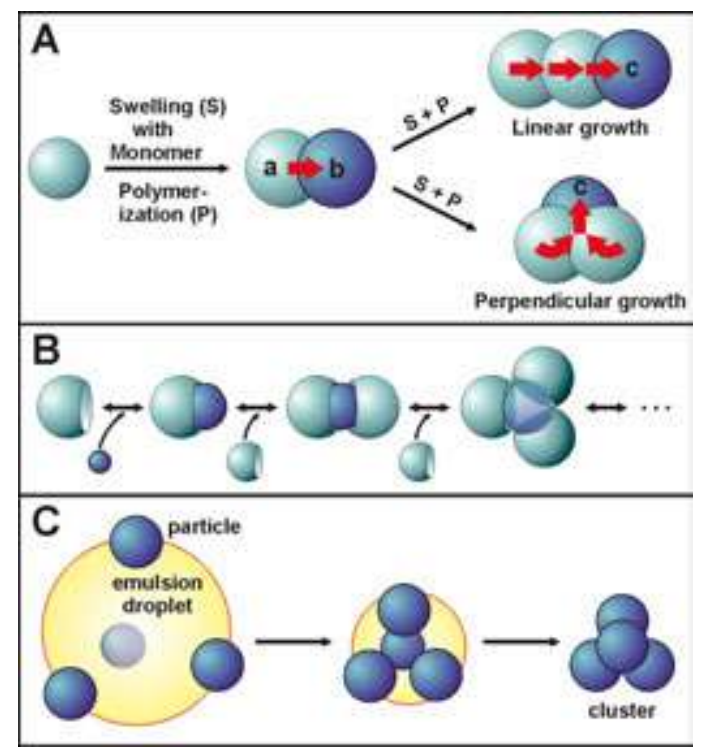

Figure 2. Routes toward "colloidal molecules": (A) Controlled phase separation during seeded emulsion polymerization: Dumbbell-shaped particles are obtained by swelling cross-linked polystyrene seeds with monomer and polymerizing it. Addition of a third bulb can follow either linear or perpendicular growth depending on cross-linking densities of bulbs a and b [3]. (B) A lock and key mechanism allows particles with complementary shapes to self-assemble into clusters in the presence of a depletant [13]. (C) Particle assembly assisted by emulsion droplets: Particles adsorb onto emulsion droplets driven by minimization of interfacial energy. Subsequent evaporation of the droplet phase forces particles to assemble into clusters, which are cemented together by van der Waals forces [15].

Chemical routes toward shape-tailored colloids are limited to specific systems and often demand careful handling of experimental parameters. Joining preformed colloidal subunits into "colloidal molecules" offers greater flexibility in the choice of elementary units. Hence, such an approach has the potential to provide access to an almost unlimited diversity of supracolloidal assemblies. Recently, first preparative strategies have been developed [1,7]. The assembly into clusters can be mediated by physical interactions, often in combination with suitable templates. A successful assembling strategy must set a limit for the aggregation. Moreover, well-defined morphologies should result. This is challenging inasmuch "colloidal atoms," unlike true atoms, usually do not undergo directed interactions. For this purpose, microspheres having oppositely charged hemispheres were prepared. These particles spontaneously self-assemble into defined clusters, which, however, are just intermediates during 
the formation of larger aggregates because there is no limitation to the size of clusters formed through electrostatic interactions [11]. Another directional assembling route is adopting Fischer's famous lock-and-key concept [12] to the colloidal regime. Site-specific recognition and association among bowl-shaped (lock) and spherical (key) colloidal subunits can be triggered by short-range depletion attractions upon adding a non-adsorbing polymer [13]. This route was successfully tested for the formation of assemblies composed of a central sphere to which a limited number of bowl-shaped colloids are bound (Figure 2B). As an alternative to geometric recognition, directional assembly via depletion interactions can be also implemented by surface roughness that locally differs [14].

It is clear that these strategies are not likely to be applied for the fabrication of "colloidal molecules" on a larger scale. Particle assembly assisted by physical templates may present an important move in this direction. In such an approach, a limited number of particles are trapped in a confined space that is given by the template. Capillary forces that occur during evaporation of the dispersion medium force the particles to pack into particle clusters. The particle assemblies are kept together by van der Waals forces. One example of this is the assembly of particles within cylindrical holes as 2-D templates [16]. Similar to the methods described above, the use of 2-D templates does not provide access to "colloidal molecules" at larger scales. Moreover, the production of submicron-sized templates for the assembly of nanoparticles and the release of the assemblies from a solid mold present further obstacles.

A promising strategy is the use of liquid droplets as 3-D templates (Figure $2 \mathrm{C}$ ). Driven by minimization of interfacial tension, particles adsorb onto emulsion droplets (Pickering effect) [17]. Subsequent evaporation of the dispersed droplet phase removes the liquid templates, while the assembly into particle clusters takes place [15]. Clusters made up of polystyrene (PS), poly(methyl methacrylate) (PMMA), or silica microspheres were prepared along these lines [18]. The supracolloids exhibit well-defined configurations, which are believed to result from an ordered arrangement of the particles at the droplet surface due to long-ranged dipole-dipole repulsion through the oil droplet and Coulomb interactions [19]. Constituents of colloidal clusters do not necessarily have to be spherical. Recently, clusters have been constructed from dumbbell-shaped particles as well [20]. This is a good example that there can be mutually complementary links between different approaches toward "colloidal molecules."

The number of particles in a given droplet determines the number of constituents of the cluster, which will result from this specific droplet. Hence, the distribution of the particles onto the droplets is directly related to the dispersity of the resulting clusters. A broad size distribution of the droplets would give rise to a broad range of different species, whereas the use of monodisperse emulsion droplets would limit the variance in the number of particles per droplet. Even monodisperse droplets do not enable the fabrication of a single set of clusters. The distribution of the particles on the droplets is random. Nonetheless, using monodisperse droplets significantly reduces the range of species and, therefore, increases the yields of distinct clusters. Some efforts have been made in this direction. If we disregard those limited to large particle assemblies [21, 22], one approach remains. In a Couette apparatus, a polydisperse precursor emulsion was transformed under shear into a narrowly dispersed emulsion bearing 
a limited number of particles on the droplets. After evaporation of the dispersed phase, high yields of particle doublets, triplets, and quadruplets were obtained [23].

Regardless of recent achievements, further efforts devoted to the fabrication of "colloidal molecules" at scales sufficient to build materials are still necessary to exploit their potential. Apart from the actual preparation, this is equally valid for sorting "colloidal molecules" into fractions of uniform configurations. If both prerequisites are satisfied, efficient strategies need to be established that allow "colloidal molecules" to assemble into well-defined superstructures.

All the above-mentioned facts indicate that properties of organized materials are strongly shaped by the dimensions of their elementary units. With regard to materials built from "colloidal molecules," the particles used for the fabrication of "colloidal molecules" represent the lowest level in hierarchy of materials (Figure 1). However, also the dimensions of the "colloidal molecules" at the middle level can be decisive, bearing already their assembly into superstructures in mind. Getting to the top level, namely to organized materials, may require "colloidal molecules" of dimensions comparable to the range of colloidal interactions allowing their assembly to proceed. This is one of the main reasons why this chapter addresses "colloidal molecules" made of nanoscale particles.

\section{Preparation of "colloidal molecules"}

The template-assisted strategies reported above are particularly suitable for the assembly of microscale particles $[7,10]$. However, microscale particles and assemblies thereof are prone to sedimentation because of their dimensions. Their motion is different from Brownian motion because there is a preferred direction dictated by gravity, which prevents random diffusion. In order to assess this, the gravitational length $l_{\mathrm{g}}$ is an appropriate measure:

$$
l_{\mathrm{g}}=\frac{D_{0}}{v_{\mathrm{s}}}=\frac{\kappa_{\mathrm{B}} T}{g \Delta \rho V_{\mathrm{p}}}
$$

where $k_{\mathrm{B}} T$ is the thermal energy, $g$ is the acceleration of gravity, $\Delta \rho$ is the difference between particle density and solvent density, and $V_{\mathrm{p}}$ is the particle volume. The gravitational length is defined as the ratio between the single-particle diffusion coefficient $D_{0}$ and the Stokes sedimentation velocity $v_{\mathrm{s}}$. It can be regarded as the minimum distance an object has to settle before the sedimentation drift exceeds the root-mean-square displacement of Brownian motion [24]. By way of illustration, the gravitational length of a polystyrene microsphere with a diameter of $1.94 \mu \mathrm{m}$ is already as short as its own diameter, whereas the gravitational length of a 100 nm-sized polystyrene particle exceeds its diameter by a factor of 141,000 .

In addition to Brownian motion acting against settling, there is a second motivation to build colloidal assemblies from nanoscale particles. Clusters from a limited number of nanoparticles have global dimensions of the same order as the range of colloidal interactions $(1-50 \mathrm{~nm})$. This 
can become an essential requirement when developing strategies, in which the assembly of "colloidal molecules" into organized superstructures is mediated by colloidal interactions [25].

Similarly to the aforementioned assembly of microspheres [15], the assembly of nanoscale elementary particles can be assisted by evaporating emulsion droplets [26, 27]. However, the diameter of the emulsion droplets has to be drastically reduced in order to allow the assembly to proceed. In addition to smaller dimensions, the droplets should also exhibit a low dispersity in size to obtain a limited number of different cluster species. This can be achieved by making use of the basic concepts behind the formation of miniemulsions [28]. The crucial point here is that the droplet size distribution in a miniemulsion can be controlled by ultrasound (Figure 3). In the following sections, all essential aspects regarding the emulsion-assisted nanoparticle assembly will be presented, starting with general considerations on the choice of the building blocks.

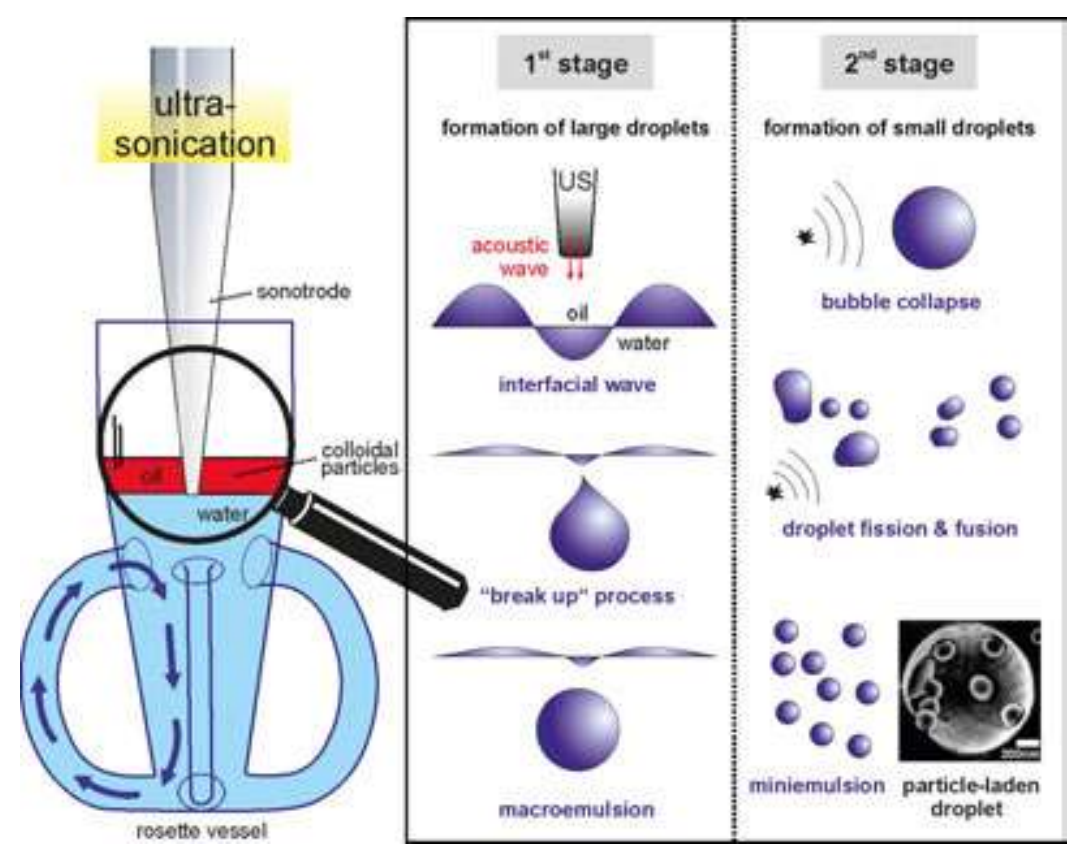

Figure 3. Schematic representation of emulsification by ultrasound: An aqueous solution containing an emulsifier is placed in a rosette vessel and overlaid with toluene. The particles to be packed into "colloidal molecules" were added either to the oil or to the water phase. The initial stage of emulsification starts with instabilities of interfacial waves that lead to eruption of the oil phase into the water phase, or vice versa, producing microscale droplets. The second stage involves fission of large droplets into even smaller ones by acoustic cavitation [36]. Collapse of cavitation bubbles creates shock waves that deform the emulsion droplets and finally cause their fission. A lower limit of the droplet size is set by droplet fusion driven by interfacial tension. The interplay of droplet fission and fusion leads to a steady state characterized by narrowly dispersed droplets. These droplets confine a certain number of particles at their surface, which marks the precondition for the preparation of "colloidal molecules" (cryo-FESEM micrograph at the lower right corner) [32]. See the text for further explanations. 


\subsection{Elementary units}

The designation "colloidal molecules" should be reserved exclusively to colloidal structures that exhibit well-defined shapes [29]. Otherwise it would not be possible to exploit their potential as building blocks with specific symmetries for hierarchically organized materials [30].

With regard to "colloidal molecules" prepared by combining ex-situ formed particles, it is thus essential to use particles that are as uniform as possible. This equally applies to the shape and the size of the particles. Generally, centrosymmetric particles are used as building blocks to avoid directional interactions in particle assembly. Hence, the configurations of the resulting assemblies are solely regulated by packing criteria for spheres, such as the formation of energetically favorable states or optimization of packing efficiencies [31, 32].

Size restrictions are also relevant to the selection of suitable building blocks. The upper size limit is set by the colloidal regime for the reasons specified above. According to the classification made by IUPAC, a colloid should have at least in one direction a dimension below $1 \mu \mathrm{m}$. If transferred to "colloidal molecules," this criterion will apply to any direction, except for linear configurations. As long as the global dimensions are maintained below $1 \mu \mathrm{m}$, Brownian diffusion prevails over gravitational forces. It is precisely because uniform particles with diameters in the range of 50-250 nm are most suitable as elementary units of "colloidal molecules." Polystyrene particles (density: $1.054 \mathrm{gm}^{-3}$ ) of such dimensions exhibit gravitational lengths that exceed their own diameter by factors between several thousands and a few millions. In this consideration, the lower limit of particle size is set by the reduced affinity of small nanoparticles to adhere to emulsion droplets. The free energy associated with the detachment $E$ of a spherical particle with radius $r$ from the oil-water interface can be as follows [33]:

$$
E=\prod r^{2} \gamma_{\text {ow }}(1-|\cos \theta|)^{2}
$$

where $\gamma_{\text {ow }}$ denotes the interfacial tension between oil and water. Eq. (2) shows that the energy needed to remove a particle from the droplet surface rapidly increases with its size. In addition, the three-phase contact angle $\theta$ marks a second key parameter when dealing with attachment or detachment of particles to emulsion droplets. It is defined by the tangents to the particle surface and the oil-water interphase in each point of the contact line where the particle and the droplet surface meet [34].

Particles having a contact angle of $90^{\circ}$ are most strongly bound to the droplet surface according to Eq. (2). They are equally wetted by oil and water because of a balance between hydrophobic and hydrophilic properties. Accordingly, ultrahydrophobic (contact angles near $0^{\circ}$ ) or ultrahydrophilic particles (contact angles near $180^{\circ}$ ) have low affinities to oil-water interphases. Hence, tuning their wetting behavior may be decisive to make particles adhere to a liquid template enabling their assembly into "colloidal molecules." Modification of the wetting behavior can be accomplished by inserting hydrophilic or hydrophobic groups into the particle surface. This can be achieved by (i) suitable comonomers during the synthesis of polymer 
colloids [27] or (ii) postmodification techniques such as silanization [19] or physisorption of amphiphiles (block copolymers, surfactants) [35]. Moreover, criteria other than the wetting behavior, such as swelling of polymer particles with the oil phase may also affect the affinity of the particles to stick to emulsion droplets [32].

Last but not least, stable suspensions of "colloidal molecules" should be obtained. In this regard, it is advantageous to use particles that bear surface charges. This yields electrostatically stabilized "colloidal molecules" without any need for post-modification [26]. Moreover, surface charges on the elementary units may be beneficial during the formation of "colloidal molecules" as well. Electrostatic repulsions among the particles support the agglomeration into energetically favorable packings and may thus contribute toward consistent configurations of "colloidal molecules" [32].

In what follows, the preparation of "colloidal molecules" built from surface-modified polystyrene and gold particles is presented. These particles meet all the above-mentioned demands. Moreover, with this selection of elementary units, it will be shown that the route toward "colloidal molecules" presented below is applicable to organic and inorganic colloids.

\subsection{Ultrasound-assisted emulsification}

The fabrication of "colloidal molecules" outlined in this chapter is based on the agglomeration of ex-situ prepared particles that are dispersed in an oil-in-water emulsion (Figure 2C). In this approach, evaporating oil droplets serve as physical templates to trap a limited number of particles and, consequently, allow their assembly into particle clusters [26]. This route toward defined particle clusters was established for the assembly of polymer and silica microspheres [18]. Droplets with diameters suitable for the assembly of microscale particles can be prepared by vigorous stirring using a rotor-stator homogenizer. However, it became apparent that the same procedure did not work out for the assembly of particles with diameters of about $100 \mathrm{~nm}$ [26]. No clusters were obtained. Most probably, the microscale droplets were split into smaller ones bearing just single particles at their surface during evaporation of the droplet phase. As a consequence, the diameters of the oil droplets had to be reduced by a comparable rate to the dimensions of the particles to be packed into clusters [27]. In addition, the dispersity of the emulsion droplets should be as as low as possible. Loading narrowly dispersed instead of polydisperse droplets by a random process will limit the range of particles on the droplets and, thus, the range of resulting assemblies [26].

Nanoparticle-laden emulsions were prepared as follows: At first, an aqueous solution of an oil-in-water emulsifier was overlaid with toluene. The elementary particles of the "colloidal molecules" were added either with the oil or the water phase. An ultrasonic horn was then dipped into the toluene until its tip touched the interphase between toluene and water, allowing propagation of ultrasound across the oil-water interphase (Figure 3). This, further assisted by the geometry of the vessel, ensured efficient mixing of the emulsion during sonication. Emulsification by ultrasound can be considered as a two-stage process, which starts with the formation of microscale droplets, which will then be split into smaller droplets with prolonged sonication [36]. The interphase between the two liquids is perturbed by ultrasound propagating along it because it acts as an acoustic boundary. As a consequence of instability 
of the interphase, microscale droplets are formed in a sort of "breakup" mechanism. At this stage, the characteristic droplet diameters are widely governed by the interfacial ultrasound wavelength and its amplitude. The droplets with dimensions of about $100 \mu \mathrm{m}$ are split into smaller ones by a mechanism that is called acoustic cavitation [36]. Above a certain threshold, sonication creates cavitation bubbles within a liquid. The bubbles grow bigger in size during rarefaction half-cycles than they shrink during compression half-cycles, resulting in an accumulative growth across the acoustic pressure cycles. On reaching a critical size, the bubbles instantaneously collapse, associated with the emission of shock waves. These, in turn, facilitate deformation of emulsion droplets and thus foster their fission into steadily smaller ones (Figure 3). Droplet fusion driven by interfacial energy sets a lower limit of the droplet size, which is characterized by a steady state among fission and fusion events. The interplay of fission and fusion yields narrowly dispersed emulsion droplets with diameters that typically fall in the range of 50-500 $\mathrm{nm}[28]$.

At the end of the ultrasonic treatment, it is important to suppress droplet growth mechanisms to preserve the average size and the narrow size distribution of the droplets. Making use of the Gibbs-Marangoni effect [37], collision growth was averted by addition of oil-in-water emulsifiers (Pluronic ${ }^{\circledR}$ F-68, Tween ${ }^{\circledR}$ 80, etc.) to the aqueous phase. Ostwald ripening, i.e., droplet growth originating from transfer of molecules among the droplets via diffusion through the continuous, favors the growth of larger droplets at the expense of smaller ones driven by the Laplace pressure [28]. Turbidity measurements revealed that Ostwald ripening was completely stopped by addition of dodecane [26, 27]. Because the hydrophobic molecules are poorly soluble in water they cannot be exchanged among the droplets. For this reason, the osmotic pressure created by the hydrophobe counteracts the Laplace pressure keeping droplet size constant.

For the preparation of "colloidal molecules," their elementary particles were either suspended in the oil or the water phase prior emulsification. Narrowly dispersed particle-laden emulsion droplets were obtained. The average sizes of the droplets were within $360-2000 \mathrm{~nm}$, depending on emulsifier concentration, stabilizing agents, and sonication conditions [27]. Cryogenic field emission scanning electron microscopy (cryo-FESEM) allowed imaging nanoparticles while confined to the droplets [32]. Their distribution at the droplet surface was random. This finding is consistent with Monte Carlo (MC) computer simulations, which indicated that nanoparticles freely diffuse at the droplet surface [32]. Cross-linked polymer particles were found to be substantially swollen at the droplet interphase if the disperse phase presents a solvent for the polymer. This could be seen by a larger diameter together with a preferential immersion into the droplet phase (Figure 3).

\subsection{Clusters of a single set of particles}

Confining a limited number of particles to droplets forms the basis for their assembly into clusters. The latter can then be accomplished by gentle evaporation of the droplet phase. Since the droplets are carrying a statistically varying number of particles, this route yields assemblies that differ in their number of constituent particles. Any experimental parameter that affects size and number of the droplets (volume and viscosity of the disperse phase, concentration of 
emulsifier or other additives, sonication conditions) is at least as important to the range of species produced as the number of elementary particles present in the emulsion [27]. Hence, optimization of experimental conditions can thus substantially help to obtain higher total yields of "colloidal molecules" or to increase the yield of distinct species. Analysis aggregation numbers and yields of clusters built from the same number of particles can be carried out by differential centrifugal sedimentation (DCS) [27]. The experimental results were in quantitative accord to predictions from MC computer simulations for a comparable set of experimental parameters [32]. DCS was used not only to explore parameters relevant to cluster formation but also to improve cluster yields.

In the case of clusters built from cross-linked polystyrene particles, the total yield could be increased to up to $74 \mathrm{wt} \%$ with regard to constituent particles [27]. Polymer particles are usually prepared by heterophase polymerizations such as emulsion polymerization [38]. Hence, the particles are already suspended in water and can be added to the emulsion via the aqueous phase. Alternatively, freeze drying and redispersion in an organic solvent permit adding them with the oil phase. It was found that the amount of clusters is considerably larger in the latter scenario [27]. This finding is specific to assemblies built from cross-linked polymer particles and is most probably related to the swelling kinetics of polymer networks. In contrast to particles that cannot swell, the adhesion to the droplet surface is not solely determined by interfacial tension. Swelling with the organic solvent may result in a preferential immersion into the oil phase. If the particles are initially suspended in the aqueous phase, they start to swell when trapped to the interface. Conversely, they are already fully swollen, if initially suspended in the oil phase and may even partially deswell when getting trapped to the oilwater interface. Hence, the kinetics of swelling or deswelling may substantially affect agglomeration into clusters, as manifested in different cluster yields [27].

Figure 4 gives an overview of cluster configurations found in experiments together with predictions from MC computer simulations. It should be noted that the polystyrene particles as elementary units of the clusters had a low degree of polydispersity. Hence, polydispersity had no influence on the experimental configurations. Indeed, the structures found in experiments were identical to the ones in simulations assuming equal-sized constituent spheres [32]. Moreover, it did not make a difference to which phases of the emulsion the particles were initially added. The latter did affect yields, but did not result in other configurations. Minimalenergy clusters of spheres have been specified for various potentials. The most prominent ones are assemblies that globally optimize the Lennard-Jones potential [39]. Configurations of clusters with aggregation numbers $A N \leq 7$ are equivalent to Lennard-Jones clusters, expect for a small number of clusters with $A N=5$ exhibiting a square pyramidal configuration. In addition, a second isomer was found for clusters with $A N=6$ at large scales in specific cases, apart from the octahedral configuration [27]. This configuration can be described as a polytetrahedral packing $\left(\mathrm{C}_{2 \mathrm{v}}\right.$ symmetry) of six spheres or, alternatively, as a pentagonal dipyramid with one particle missing. The configuration presents a minimal-energy packing of hard spheres [31], but was not found in computer simulations mimicking the droplet-based assembly route [32]. 
While packing efficiency is prevalent for small $A N$, the assembly route itself can have an influence on the configurations. The assembly proceeds at the surface of a spherical template that is concomitantly shrinking and has no particles inside (Figure 2C). Hence, a constraint originating from this strategy may hamper mere optimization of packing efficiency. In fact, predominant configurations were found for $8 \leq A N \leq 12$, both in experiments and simulations [32]. Packing of an equal number of hard spheres would permit more minimal-energy structures [31]. However, particle clusters templated by evaporating emulsion droplets did not exhibit a corresponding range of isomers, no matter whether built from nanoscale [27] or microscale particles [15]. This emphasizes that the template takes an active role when the "colloidal molecules" develop their final configurations [32].

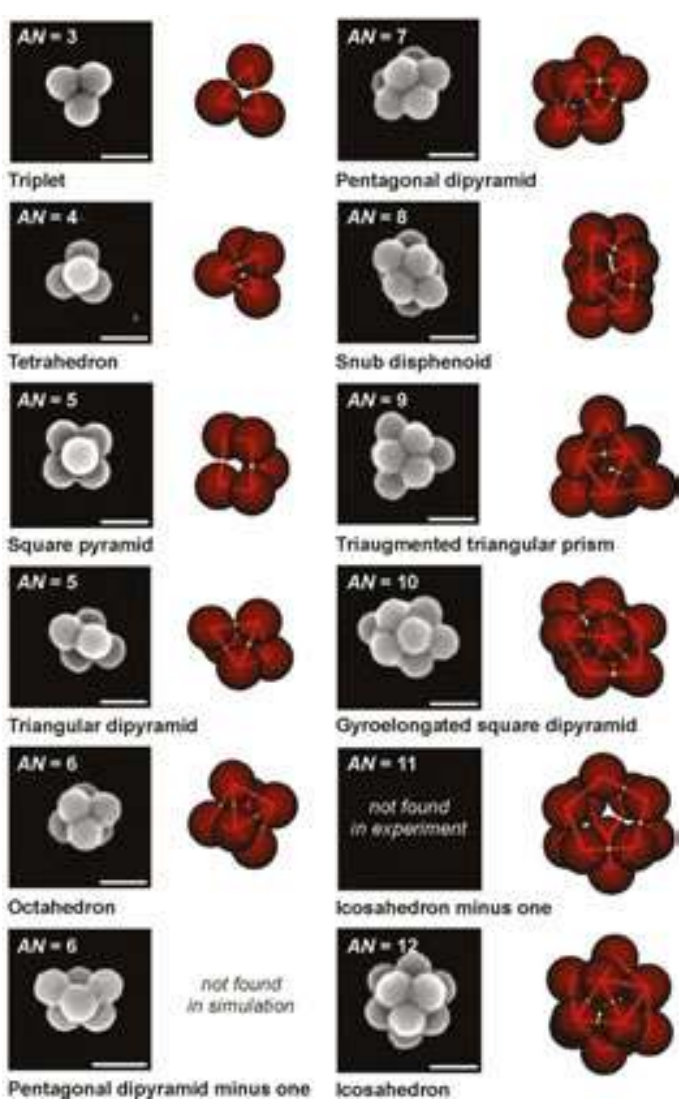

Figure 4. Joining polystyrene particles (average diameter: $154 \mathrm{~nm}$ ) on droplets led to clusters with unique configurations, primarily characterized by the aggregation numbers AN. Cluster morphologies found in FESEM micrographs (left columns) agree with those found in MC computer simulations (right columns). The wireframe in the structures from simulations visualizes the geometric arrangement of the particle centers. The scale bars indicate $200 \mathrm{~nm}$. Reproduced with permission from Ref. [32]. (C) 2011, AIP Publishing LLC. 
The droplet-assisted strategy is not limited to polymer particles. It can be applied to the assembly of inorganic particles as well [35]. Nevertheless, there is a notable difference. Unlike polymer particles, inorganic particles do not swell. It would thus require hydrophobic particles to get them dispersed in the oil phase. In doing so, hydrophobic "colloidal molecules" that immediately self-assemble into large fractal structures would result. It is therefore advantageous to use inorganic particles that can be dispersed in the aqueous phase. Compatibility to water can be improved by surface coatings that make the particles more hydrophilic. It should be noted here, however, that the contact angles of the particles should not become too low to make them still adhere to the droplet surface (see Eq. (2)). Gold nanoparticles bearing a polymer coating were found to perfectly meet this requirement [35]. The conceptual expansion to "colloidal molecules" built from gold particles is motivated by intense plasmonic "hot spots" that may occur within such structures [40].

Gold nanoparticles were prepared by a kinetically controlled seed-mediated growth method in the presence of cetyltrimethylammonium bromide (CTAB) as a surfactant [41]. The multifaceted particles with average diameters of up to $70 \mathrm{~nm}$ exhibited low dispersities in size and shape, which is essential for the fabrication of "colloidal molecules." The CTAB molecules were exchanged by the commercial block copolymer (Pluronic ${ }^{\circledR}$ F-68) by repeated centrifugation and redispersion processes. The exchange of the surface layer was accompanied by a change of the zeta potential, which was used to check completeness of this process [35]. The polymer coating onto the particles was a key element for fabricating assemblies with intense plasmonic "hot spots."

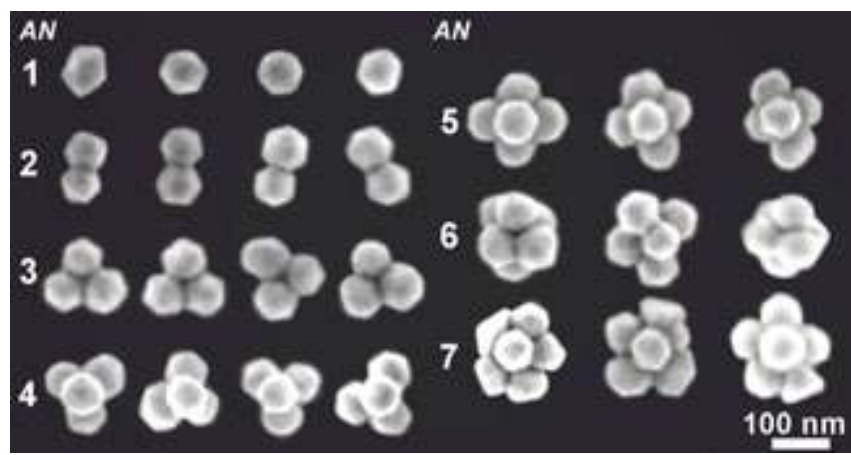

Figure 5. Configurations of clusters built from variable numbers $(A N)$ of polymer-coated gold nanoparticles [35].

The assembly of polymer-coated gold nanoparticles was accomplished along similar lines to polymer particles. During emulsification by ultrasound, the disperse phase is broken down in a large number of small droplets. The volume of the dispersed toluene was thus kept as low as possible in view of the fact that such gold particles are available in limited quantities. This ensured that there were sufficient toluene droplets carrying two or more gold nanoparticles, allowing cluster formation to proceed. Evaporation of the droplet phase produced clusters of two to seven polymer-coated nanoparticles using the emulsion droplet-based technique 
described above [35]. As expected, shapes of "colloidal molecules" built from faceted particles were less regular than that built from amorphous spheres. Nonetheless, the plasmonic clusters had defined configurations (Figure 5). Applications of plasmonic "colloidal molecules" in surface-enhanced spectroscopies will be discussed in Section 3.2.

\subsection{From hybrids to capsules}

The range of accessible "colloidal molecules" can be further extended when different sets of particles are combined. The adsorption of nanoparticles onto ex-situ formed particle clusters (Figure 6A) imparted by electrostatic interactions is a straightforward route in this direction [42]. Long-term stable suspensions of composite particles were obtained at low and high surface coverage. Flocculation occurs near the isoelectric point of the composites, when surface charges of the support are balanced by the ones on the adsorbed nanoparticles. The versatility of the route was demonstrated for inorganic nanoparticles that differ in their chemical nature, size and surface charge [42]. Random sequential adsorption superimposed by electrostatic repulsions governs the positioning of the nanoparticles yielding spatially separated nanoparticles on the colloidal support (Figure 6B).

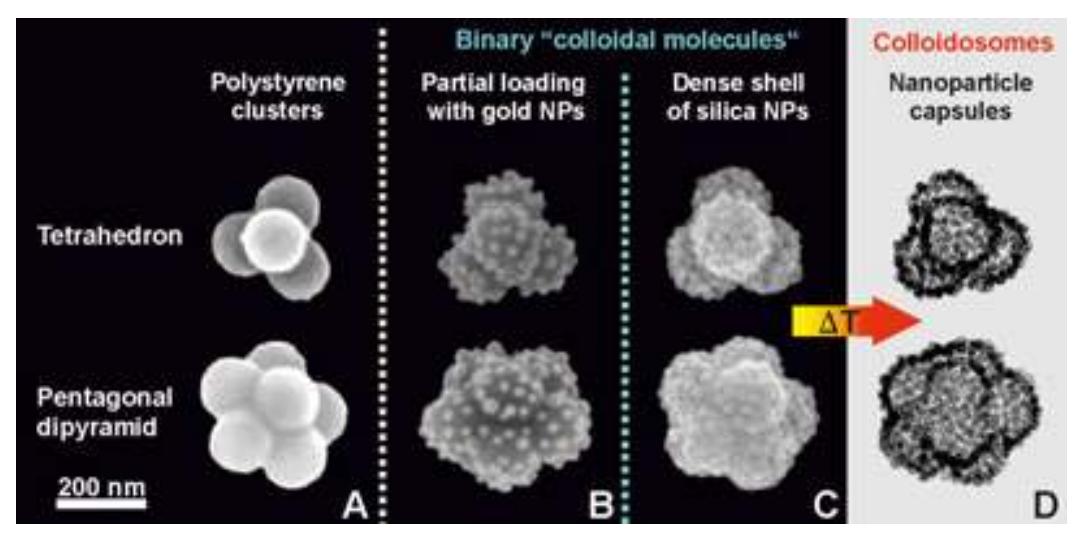

Figure 6. Formation of binary "colloidal molecules": Physisorption of negatively charged nanoparticles onto preformed clusters of positively charged polymer particles $(\mathrm{A} \rightarrow \mathrm{B})$ [42]; co-assembly of polymer particles and oppositely charged nanoparticles at the surface of emulsion droplets yields core-shell heteroaggregates (C) that can act as precursors for the preparation of nanoparticle capsules with complex shapes (D) [43]. See the text for further explanations.

Dense nanoparticle shells on particle clusters were accessible by another strategy, which was based on the emulsion-assisted process [43]. In this approach, cross-linked polystyrene particles were co-assembled with oppositely charged silica nanoparticles, with the polymer particles added via the dispersed (toluene) phase, whereas the nanoparticles were suspended in the continuous (water) phase of the emulsion. This procedure allows oppositely charged particles to co-assemble at the oil-water interphase, while avoiding the formation of fractal aggregates. 
Minimization of interfacial energy facilitates confinement of the larger polystyrene particles to the droplet surface. The affinity of silica nanoparticles to stick to the interface is a lot weaker due to their smaller dimensions (see Eq. (2)). This favored their deposition onto the surface of the oppositely charged polymer particles, evidently to the sides that were exposed to the continuous water phase. During adsorption of the nanoparticles, the polymer particles were substantially swollen with the dispersed toluene. Evaporation of the dispersed toluene induced deswelling of the polymer particles, and in parallel to this, their assembly into clusters. Deswelling of their support brought the nanoparticles closely together irrespective of electrostatic repulsions. MC computer simulations revealed that, given an appropriate number of nanoparticles per polymer particle, a dense shell of nanoparticles is formed tightly encasing a core of aggregated polymer spheres [43]. This is in agreement with experimental observations. The global shapes of the binary heteroaggregates were precisely determined by the number of polymer spheres forming the core (Figure 6C). As a result, the global shapes of the binary "colloidal molecules" were identical to aggregates built from a single set of particles.

The core-shell-type assemblies could be transferred into nanoporous capsules, simply by calcination at $500^{\circ} \mathrm{C}$ (air atmosphere). The polymer was completely removed during thermal treatment, as confirmed by elemental analysis [43]. Shaping the inner cavity by polymer particles yields compartmentalized nanoporous capsules (Figure 6D). Remarkably, all nanoparticle capsules were intact after the decomposition of the core despite they consist of a single layer of nanoparticles kept together by nanoscopic contacts. Hence, the capsules neither burst nor evaporate over the calcination. MC computer simulations revealed that interplay of short-range (van der Waals) attractions and long-range (electrostatic) repulsions can trap each nanoparticle in a local energy minimum [43]. Moreover, tiny bridges among the nanoparticles may form during calcination by the condensation of silanol groups at the silica surface.

Template-based assembling strategies can be effective tools for the preparation of complex supraparticles, which can be further used as precursors for tailor-made colloids. In this specific case, it gave access to a unique class of colloidosomes built from a single layer of nanoparticles. They are distinguished from other colloidosomes by shapes that are complex but still welldefined and have a high density of nanopores (Figure 6D). Such nanoparticle capsules might find applications as model systems for biological cells or as drug delivery vehicles.

\subsection{Sorting "colloidal molecules"}

In order to use "colloidal molecules" as buildings blocks for novel materials that require particles with specific symmetries or high complexity, it is crucial to have them sorted into uniform species. Sorting can be accomplished by density gradient ultracentrifugation (Figure 7) [26]. Rate zonal (density gradient) ultracentrifugation separates particle clusters according to their number of constituents, or more fundamentally by their sedimentation coefficients. This technique enables efficient sorting of "colloidal molecules" owing to high resolution because assemblies whose sedimentation coefficients differ by as small as $15 \%$ can be separated [44]. Besides superior resolving power, it allows sorting in suspension at a minimum of undesirable wall effects. In contrast to isopycnic centrifugation, which separates particles according to their densities, the role of density gradients in rate zonal centrifugation 
is secondary, but nevertheless necessary. It is used to eliminate currents (streaming) that have an adverse effect on banding particles according to their sedimentation rates in defined zones. Zonal centrifugation requires rotors, in which the density gradient is always parallel to the force onto which it is subjected [44]. This can be ensured by using high-speed swinging bucket rotors, which allowed efficient separation of particle clusters in scales that are sufficient for analytical purposes or practical applications that do not require large amounts [45]. Specific examples will be introduced and discussed in the following section.

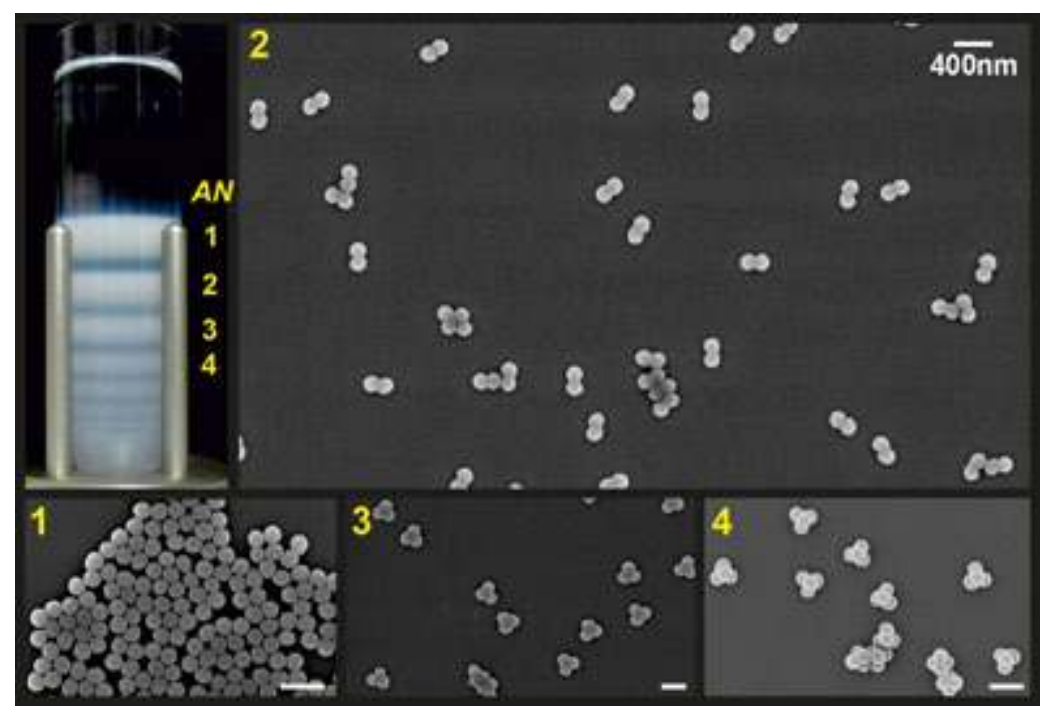

Figure 7. Sorting of submicron-sized clusters of polystyrene particles was accomplished by density gradient centrifugation using a high-speed swinging bucket rotor (photograph). Fractionation according to their sedimentation coefficients resulted in suspensions that contain an equal number of constituents: (1) single particles, (2) particle dimers, (3) particle trimers, and (4) particle tetramers. The scale bars in the FESEM micrographs indicate $400 \mathrm{~nm}$.

\section{Specific applications}

\subsection{Model colloids}

The diffusion of colloids with complex shapes is a key to a comprehensive understanding of practical phenomena such as biodistribution, sedimentation, coagulation, floatation, and rheology [46]. While the diffusion of particles with simple shapes such as spheres [47, 48], ellipsoids [49, 50], rods [51, 52], and platelets [53] was extensively studied by experiment [54], simulation [55], and theory [56]; studies on the diffusion of complex colloids are still quite scarce [57]. In this context, their defined geometries make "colloidal molecules" ideal model systems to explore the motion of colloidal objects with arbitrary shapes. This approach was introduced by Granick and co-workers using planar clusters of microspheres, which can be 
easily analyzed by optical microscopy. The motion of microscale objects is, however, affected by gravitational forces because of their rather small gravitational lengths (see Section 2). Moreover, analyzing particles near a solid wall, in the form of a microscope slide, can substantially change the diffusion coefficients [58]. These problems can be elegantly avoided if (i) the size of the clusters is maintained in the submicron regime with Brownian dynamics prevailing over gravitational forces, and if (ii) particle motion is studied within the bulk phase. The latter can be accomplished by light scattering techniques that allow studying the motion of shape-anisotropic particles over relevant length scales.
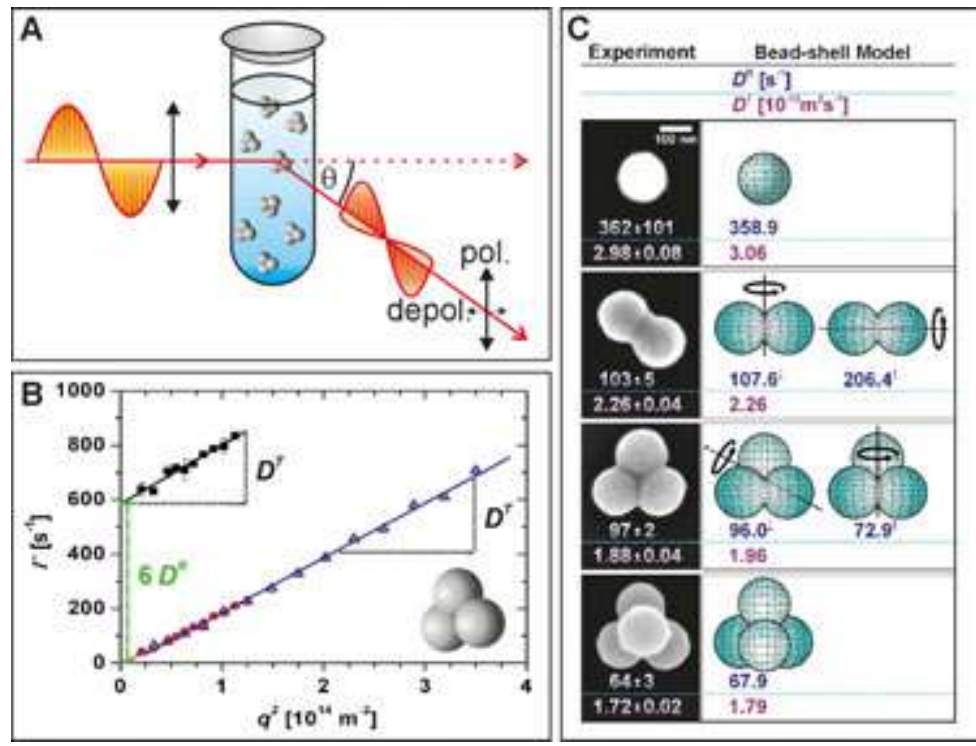

Figure 8. Diffusion of "colloidal molecules": Schematic representation of depolarized dynamic light scattering (DDLS); a horizontally oriented polarizer is used to measure the depolarized component of the scattered light, which provides insights into the translational and rotational diffusion of "colloidal molecules" (A). Relaxation rates $\Gamma$ as the function of the square of the absolute values of the scattering vector $q$ follow linear correlations that provide access to the translational $\left(D_{\mathrm{T}}\right)$ and rotational $\left(D_{\mathrm{R}}\right)$ diffusion coefficients $(\mathrm{B})$. The fast mode is represented as black squares; the slow mode of DDLS (red spheres) agrees with results obtained from polarized DLS (blue triangles). Synopsis of experimental and theoretical diffusion coefficients $(C)$ : Underneath the experimental clusters that are oriented with their main body parallel to the plane of the figure, $D_{\mathrm{T}}$ and $D_{\mathrm{R}}$ as obtained by DDLS are given. These values agree well with theoretical predications for $D_{\mathrm{T}}$ and values for $D_{\mathrm{R}}$ perpendicular and parallel to the main symmetry axis using the bead-shell model [62], which considers the exact geometries of the clusters. Reproduced with permission from Ref. [45]. (C) 2009, American Chemical Society.

Dynamic light scattering (DLS) is the most common technique to probe the diffusion of colloidal particles $[59,60]$. In the case of optically isotropic particles, decorrelation of scattered light is solely due to translational diffusion and thus cannot provide any insights into rotational motion. However, colloidal particles that are optically anisotropic in shape or composition may produce different orientations of incident and scattered electromagnetic fields. Hence, contributions of scattered light polarized in two directions perpendicular to each other can be 
detected using a polarizer setup [61]. It should be noted that the intensity of the scattered light that is polarized perpendicular to the intensity of the incident light is related to the meansquared optical anisotropy of the particles and can be thus rather poor for colloids with low aspect ratios [51]. However, this mode providing insights into translational and rotational diffusion becomes accessible by depolarized dynamic light scattering (DDLS), which is based on a polarizer and analyzer principle (Figure $\mathbf{8 A}$ ).

DDLS was used to probe rotational and translational dynamics of "colloidal molecules" that differ in their geometries defined by the aggregation number. The study was limited to fractions of clusters of not more than four $154 \mathrm{~nm}$-sized polystyrene particles because such assemblies exhibited unique configurations if prepared by the droplet-assisted route (Figure 4). Rate-zonal density gradient centrifugation made the clusters available in sufficient quantities and sorting accuracy to measure well-defined intensity autocorrelation functions [45].

In the DDLS experiments, the depolarized autocorrelation functions (an analyzer perpendicular to a polarizer) were found to be the sum of two discrete exponential decays, whereas the polarized intensity autocorrelation functions (an analyzer and a polarizer aligned to each other) were consistent with single exponential decays [45]. Hence, two discrete modes associated with the motion of the clusters were observed in the depolarized analyzer-polarizer setup (Figure 8A). The slow mode of the depolarized experiment was equivalent to the single mode of the polarized experiment (Figure 8B). Moreover, the decay rates were in agreement with standard DLS experiments (in the absence of an analyzer). Hence, the slow mode of the depolarized is solely associated with the translation of the clusters and results from the limited extinction ratio of the analyzer. This yields a small portion of scattered light that is polarized equal to the incident light, which is in agreement with a standard DLS experiment, providing insights into translational dynamics. Accordingly, the fast mode represents the true depolarized signal. Its decay rate is associated with translational and rotational dynamics providing access to translational and rotational diffusion coefficients (Figure 8B). Simple scaling laws were applicable pointing to decoupling rotational and translational motion, which might be an immediate consequence of the small dimensions of the clusters [57]. In contrast to microscale particles, submicron-sized particles may travel distances equal to a multitude of their own diameter in bare translation movements before they undergo directional changes caused by rotation. Decoupling of rotational and translational motion produces linear scaling laws if the decay rates are plotted against the square of the absolute value of the scattering vector facilitating data analysis and making submicron-sized "colloidal molecules" ideal candidates to probe the diffusion of complex particles [45].

Interestingly, it turned out that even the spherical elementary units of the particle clusters produced a poor depolarized signal despite the fact that they should be almost optically isotropic in shape and composition. Nevertheless, the signal provided access to the rotational diffusion coefficient of the elementary units of the clusters, and thus gave a useful reference to the evaluation of the corresponding values derived for the clusters. In line with the correlation of the depolarized intensity to the optical anisotropy, the depolarized signal was strongest for 
dimer particles, whereas it was substantially lower for trimers and tetramers because of the concomitant decrease in the aspect ratio with the increasing aggregation number [45].

The translational diffusion was found to be widely governed by the global dimensions of the "colloidal molecules." This is primarily expressed by the observation that the translational diffusion coefficients are approximately governed by the reciprocal value of the average dimensions of the "colloidal molecules," in rough agreement with the Stokes-Einstein equation for spherical particles [59]. In contrast to translational motion, shape plays a prominent role in rotational dynamics. This is particularly visible in similar rotational diffusion coefficients of dimer and trimer particles, irrespective of dissimilar spatial dimensions (Figure 8C). DDLS is sensitive to the rotation of an object around the axis perpendicular to its main symmetry axis, whereas it cannot monitor the rotation around its main symmetry. This explains why the rotational diffusion coefficients of dimer and trimer particles are similar. The volume displaced by a rotation around the axis perpendicular to the main symmetry axis is equal for a dimer and trimer particle.

Further interpretation of the diffusion of "colloidal molecules" can be based on hydrodynamic modeling. Garcia de la Torre and co-workers established a bead-shell modeling strategy that is perfectly applicable to "colloidal molecules" $[62,63]$. In this model, the shape of the "colloidal molecules" is mimicked by overlapping spheres. The surface exposed to the surrounding liquid is represented by a shell of touching but not overlapping small beads that act as friction elements. Hydrodynamic properties were calculated using such a treatment for various types of "colloidal molecules." The predictions for the rotational and translational diffusion coefficients were in perfect agreement with the experimental results (Figure 8C). This was achieved by assuming stick boundary conditions, whereas treatments based on slip boundary conditions did not reproduce the experimental results [45]. Substantially, lower orientation times of colloidal particles as compared to molecules make stick boundary conditions favorable over slip boundary conditions.

The study has demonstrated that "colloidal molecules" can act as models for particles with complex shapes. Understanding their diffusion can also contribute to gain deeper insights into the formation of matter because particle clusters are intermediates during the assembly of individual particles into macroscale structures.

\subsection{Substrates for surface-enhanced spectroscopies}

Beyond their use as model colloids, "colloidal molecules" may find other applications because of specific properties that emerge when nanoparticles are arranged in close configurations. Properties of densely packed nanoparticles are often quite distinct from properties of spatially isolated particles. It is thus of practical and fundamental importance to produce organized nanoparticle assemblies and make their collective properties, such as plasmon coupling, magnetic coupling, resonance energy transfer enhanced photoconductivity, available for applications [64]. An example of this is the surface-enhanced Raman scattering, which can be several orders in magnitude higher for nanoparticle assemblies than for single gold nanoparticles $[35,40,65]$. 
Raman spectroscopy is based on inelastic scattering of monochromatic light. It provides rich information on structure and composition of matter based on its vibrational modes. However, the Raman effect itself is rather poor. As for comparison, typical Raman cross-sections can be up to 14 orders in magnitude lower than relevant cross-sections in fluorescence spectroscopy [66]. For this reason, the invention of the laser marked an important milestone in the establishment of Raman spectroscopy in analytical laboratories. A comparable revolutionary coup might be based on the finding that if the analyte molecules are bound to plasmonic nanostructures, a large enhancement of the Raman stock signal will result [40]. Surface-enhanced Raman scattering (SERS) can be attributed to two different mechanisms. Firstly, strong magnifications of the fields of the incident light and the Raman scattered light result from interactions among the two optical fields and surface plasmons localized on the plasmonic substrate. Secondly, the electronic interaction among analyte molecules and the metallic substrate can itself modify the scattering process and produce efficiently larger Raman crosssections [66]. The magnitude of signal enhancement that can be achieved is considerably influenced by the shape of the SERS substrate. This is supported by recent calculations of enhancement factor distributions along the surface of silver nanoparticles of distinct geometries [67]. A $30 \mathrm{~nm}$-sized silver sphere has two rather broad plasmonic "hot spots" (i.e. location where the enhancement reaches a maximum) at the poles. At these sites a signal enhancement of five orders in magnitude is found. Over the entire sphere, the Raman signal is still four orders in magnitude higher than in the absence of a plasmonic substrate. A comparable ellipsoidal SERS substrate shows two distinct hot spots exactly at its tips, providing SERS enhancement of already seven orders in magnitude. In the case of two silver nanoparticles separated by a gap of $2 \mathrm{~nm}$, an extraordinary signal enhancement of nine orders in magnitude is predicted [67]. This example clearly indicates that clusters of plasmonic nanoparticles are promising SERS substrates because they may pool several hot spots within one colloidal object. To produce intense hot spots, it is necessary to accurately adjust the interparticle distances by suitable spacers. Gap engineering is thus crucial to the production of efficient SERS substrates. On the one side, the gap dimensions should be not too large to produce hot spots and, on the other side, they must be large enough to provide access for analyte molecules. Based on the above-mentioned calculations, a gap size of $2 \mathrm{~nm}$ can be considered as a well-balanced compromise between the two contrary requirements.

High-resolution transmission electron microscopy (HRTEM) images (Figure 9) demonstrate that such interparticle distances are found for the clusters of polymer-coated gold nanoparticles described in Section 2.3. The use of a synthetic polymer coating for creating the required interparticle gaps has several advantages as compared to DNA, thiolated or covalent linkers. It allows efficient linking of colloidal particles with nanoscale interparticle distances [40, 65]. It also allows analyte molecules diffusing into the gap because of the low volume fraction of the polymer and, in specific cases, even concentrating there [68, 69]. Most importantly, polymers have low Raman cross-sections [70]. This avoids considerable falsifications of the vibrational signals by the gap material.

In the light of the above, "colloidal molecules" built from $50 \mathrm{~nm}$-sized gold particles with 2 $\mathrm{nm}$ interparticle gaps created by a polymer coating combine high optical coupling with strong 
electromagnetic fields generated from particles of such diameters. The SERS enhancement was directly measured for clusters made up from 2 to 7 individual gold nanoparticles [35]. To this end, highly diluted suspensions of the assemblies were spin-coated on a microscope slide bearing a rectangular-coordinate grid pattern. This ensured sufficient spatial separation among the clusters and the retrieval of specific species allowing studies at the level of single plasmonic "colloidal molecules." Areas containing an isolated cluster were studied first by FESEM, then by dark-field optical microspectroscopy, and finally, by high-resolution SERS after adsorption of benzenethiol from the gas phase [35]. This procedure helped to determine configuration, optical response, and SERS enhancement of the very same plasmonic "colloidal molecule."

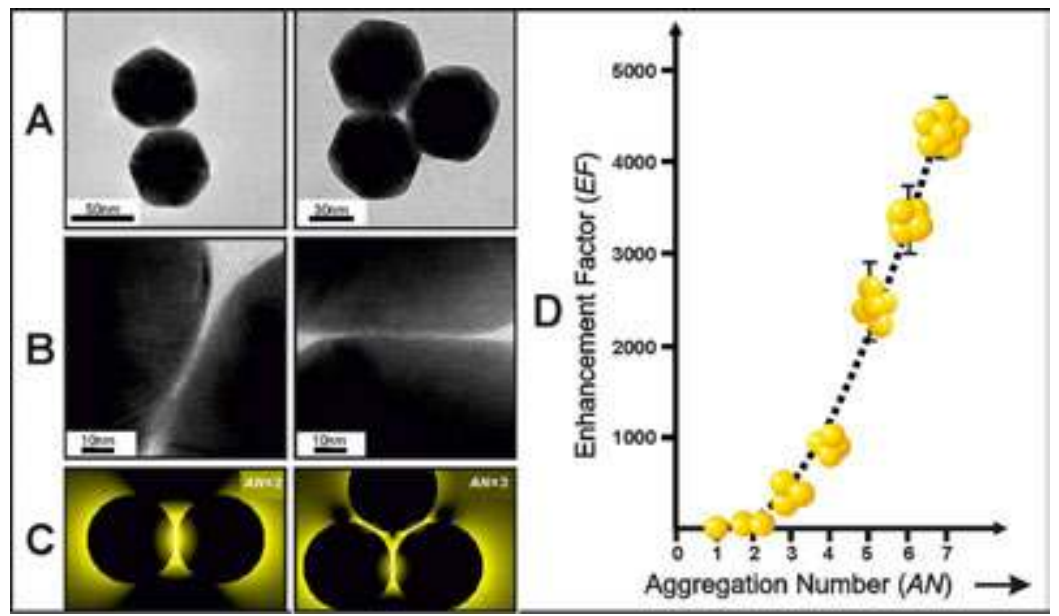

Figure 9. Plasmonic "colloidal molecules": HRTEM micrographs of gold nanoparticle dimers and trimers illustrating the $2 \mathrm{~nm}$-sized interparticle gaps (A, B). Near-field intensities on a log thermal scale for dimer and trimer clusters along the plane passing through the particle centers showing the occurrence of intensive plasmonic "hot spots" right at the interparticle gaps (C). Optical enhancement factors that are achieved by combining single nanoparticles into clusters, normalized to the enhancement observed for a number of individual particles equal to the aggregation numbers (D).

Detailed information on the experimental optical response together with electromagnetic simulations can be found in Ref. [35]. At this place, the actual SERS enhancement that is achieved with specific cluster configurations shall be discussed. This section should be read bearing in mind that the Raman signals using single gold nanoparticles, that is to say, using the elementary units of the clusters as SERS substrates, are already five orders in magnitude higher than in the absence of any plasmonic colloids. If, however, the plasmonic nanoparticles are assembled into clusters of a rather small number of particles, the signal dramatically increases up to a level of three orders in magnitude (Figure 9). Even under off-resonant illumination conditions, a dimer particle can provide an intensity increase close to a 100 -fold. Particles packed into a pentagonal bipyramid reach 5000-fold additional SERS enhancement. Interestingly, a simple scaling law can be used as a reference to predict the optical enhance- 
ment factors $E F$ as a function of the aggregation number $A N$, and, related to this, the number of interparticle gaps $G N$ :

$$
E F=A N+A^{*} G N
$$

Hence, the actual SERS enhancement is mainly governed by the number of interparticle gaps. This is fully understood when near-field distributions around the clusters are taken into account (Figure 9). They show intense plasmonic "hot spots" right at the gaps, which are almost exclusively responsible for the extraordinary enhancement, whereas other regions of the supracolloidal SERS substrate hardly contribute [35]. For this reason, the prefactor $A$ in Eq. (3) is virtually identical to the contribution of a single plasmonic hot spot. Indeed it is found that this contribution is almost independent of the aggregation number of the substrate [35]. This clearly indicates that the hot spots form a regular pattern within the highly symmetric plasmonic "colloidal molecules," and act almost independently. It is evident that an even higher SERS enhancement may be achieved if clusters of higher aggregation numbers are used. However, larger assemblies of gold nanoparticles exhibit less regular configurations and are thus less suitable as model SERS substrates.

In light of their high SERS enhancements and their facile preparation, plasmonic "colloidal molecules" may find applications in the routine analytics of minute quantities of analyte molecules.

\section{Conclusions}

The results have shown that it is possible to build "colloidal molecules" even from nanoscale particles. Precise configurations are accessible given that the elementary particles have about the same size and shape. The preparation can be based on confining particles to evaporating emulsion droplets. Using ultrasound for emulsification provides control on droplet sizes and dispersity, which is an absolute prerequisite to the production of "colloidal molecules" from nanoscale building blocks. The emulsion-based route is quite versatile. It can be both applied to organic and inorganic particles. In both cases, assemblies with defined configurations can be obtained. Moreover, different sets of particles can be combined into binary supracolloids. It is also possible to co-assemble particles at the droplet surface that came into the emulsion via different phases.

Given that they are built from nanoparticles, "colloidal molecules" have dimensions of less than one micrometer. Consequently, they underlie Brownian motion, which prevails over sedimentation. Moreover, the diffusion of submicron-sized "colloidal molecules" can be studied by scattering techniques. This, combined with the knowledge of their precise geometry, makes clusters of nanoparticles to excellent model colloids aiming at a general understanding of the diffusion of particles with complex shapes, such as protein molecules. DDLS gave direct insights into the diffusion of "colloidal molecules" in dilute suspensions. Relationships for translational and rotational motion were identified for particle geometries, which cannot be 
described by means of the Stokes-Einstein equation for spherical particles. Knowing the geometry of the anisotropic colloids, the experimental diffusion coefficients could be compared to predictions from hydrodynamic models.

Beyond their use as model systems, "colloidal molecules" offer other exiting perspectives. Unique physical properties can emerge from combining functional subunits into assemblies with tailored shapes and symmetries. The occurrence of plasmonic hotspots in gold nanoparticle clusters is a clear example of a property that is not found in particles that are spatially separated from each other. Signal enhancements exceeding the ones achieved with single particles are found if plasmonic "colloidal molecules" are used as substrates in surfaceenhanced spectroscopies. Moreover, varying the surface coating on the elementary units can be used to modify the plasmonic properties. In the course of further advancements with regard to plasmonic "colloidal molecules," more applications in sensing and nonlinear nanophotonics can be expected.

"Colloidal molecules" are particularly promising with regard to innovative applications that demand materials with specific symmetries or functionality. The anisotropic nature of "colloidal molecules" due to their non-spherical shapes could be exploited to their selfassembly into the next higher level of hierarchically organized materials. To this end, interactions among "colloidal molecules" need to be sufficiently weak but long-ranged and specific in order to allow the formation of close-packed equilibrium phases to proceed. Gelation, which prevents the formation of ordered assemblies, can be suppressed by external fields due to the anisotropic polarizability of non-spherical colloids. This technique was successfully applied to produce long-range ordered photonic crystals from dumbbell-shaped "colloidal molecules" [4]. Future efforts must be geared to giving "colloidal molecules" valences suitable for their directed assembly. A recent study about the spontaneous assembly of microspheres bearing two attractive patches at specific positions into well-defined Kagome-like structures is pointing in this direction [8].

Another challenge to be tackled is to equip "colloidal molecules" with tunable interparticle distances mimicking molecular flexibility. This would further contribute to bringing "colloidal molecules" closer to true molecules. Hierarchically organized materials made of such "colloidal molecules" could exhibit stimuli-regulated properties, such as self-healing, defect minimization, or switchable optical properties that are induced by changes in size and configuration.

\section{Acknowledgements}

Financial support from the Deutsche Forschungsgemeinschaft (DFG) within SFB 1214/A10 and the Struktur- und Inovationsfonds für die Forschung in Baden-Württemberg (SI-BW) is gratefully acknowledged. 


\section{Author details}

Claudia Simone Plüisch and Alexander Wittemann*

*Address all correspondence to: alexander.wittemann@uni-konstanz.de

Colloid Chemistry, Department of Chemistry, University of Konstanz, Konstanz, Germany

\section{References}

[1] Sacanna S, Korpics M, Rodriguez K, Colón-Meléndez L, Kim S-H, Pine DJ, Yi GR. Shaping colloids for self-assembly. Nat Commun. 2013;4:1-6. DOI: 10.1038/ ncomms 2694

[2] Ye XC, Jin LH, Caglayan H, Chen J, Xing GZ, Zheng C, Doan-Nguyen V, Kang YJ, Engheta N, Kagan CR, Murray CB. Improved size-tunable synthesis of monodisperse gold nanorods through the use of aromatic additives. ACS Nano. 2012;6:2804-2817. DOI: $10.1021 / \mathrm{nn} 300315 \mathrm{j}$

[3] Kim JW, Larsen RJ, Weitz DA. Uniform nonspherical colloidal particles with tunable shapes. Adv Mater. 2007;19:2005-2009. DOI: 10.1002/adma.200602345

[4] Forster JD, Park JG, Mittal M, Noh H, Schreck CF, O'Hern CS, Cao H, Furst EM, Dufresne ER. Assembly of optical-scale dumbbells into dense photonic crystals. ACS Nano. 2011;5:6695-6700. DOI: 10.1021/nn202227f

[5] Taveau J-C, Nguyen D, Perro A, Ravaine S, Duguet E, Lambert O. New insights into the nucleation and growth of ps nodules on silica nanoparticles by $3 \mathrm{~d}$ cryo-electron tomography. Soft Matter. 2008;4:311-315. DOI: 10.1039/B710137F

[6] van Blaaderen A. Materials science: Colloids get complex. Nature. 2006;439:545-546. DOI: $10.1038 / 439545 a$

[7] Duguet E, Desert A, Perro A, Ravaine S. Design and elaboration of colloidal molecules: An overview. Chem Soc Rev. 2011;40:941-960. DOI: 10.1039/c0cs00048e

[8] Chen Q, Bae SC, Granick S. Directed self-assembly of a colloidal kagome lattice. Nature. 2011;469:381-384. DOI: 10.1038/nature09713

[9] Yan J, Bae SC, Granick S. Colloidal superstructures programmed into magnetic janus particles. Adv Mater. 2015;27:874-879. DOI: 10.1002/adma.201403857

[10] Sacanna S, Pine DJ. Shape-anisotropic colloids: Building blocks for complex assemblies. Curr Opin Colloid Interf Sci. 2011;16:96-105. DOI: 10.1016/j.cocis.2011.01.003

[11] Hong L, Cacciuto A, Luijten E, Granick S. Clusters of charged janus spheres. Nano Lett. 2006;6:2510-2514. DOI: 10.1021/n1061857i 
[12] Steed JW, Turner DR, Wallace KJ, editors. Core concepts in supramolecular chemistry and nanochemistry. Chichester: John Wiley \& Sons Ltd.; 2007. 307 p. ISBN: 978-0-470$85867-7$

[13] Sacanna S, Irvine WTM, Chaikin PM, Pine DJ. Lock and key colloids. Nature. 2010;464:575-578. DOI: 10.1038/nature08906

[14] Kraft DJ, Ni R, Smallenburg F, Hermes M, Yoon K, Weitz DA, van Blaaderen A, Groenewold J, Dijkstra M, Kegel WK. Surface roughness directed self-assembly of patchy particles into colloidal micelles. Proc Natl Acad Sci. 2012;109:10787-10792. DOI: 10.1073/pnas.1116820109

[15] Manoharan VN, Elsesser MT, Pine DJ. Dense packing and symmetry in small clusters of microspheres. Science. 2003;301:483-487. DOI: 10.1126/science.1086189

[16] Xia YN, Yin YD, Lu Y, McLellan J. Template-assisted self-assembly of spherical colloids into complex and controllable structures. Adv Funct Mater. 2003;13:907-918. DOI: 10.1002/adfm.200300002

[17] Pieranski P. Two-dimensional interfacial colloidal crystals. Phys Rev Lett. 1980;45:569_ 572. DOI: 10.1103/PhysRevLett.45.569

[18] Yi G-R, Manoharan VN, Michel E, Elsesser MT, Yang S-M, Pine DJ. Colloidal clusters of silica or polymer microspheres. Adv Mater. 2004;16:1204-1208. DOI: 10.1002/ adma. 200306638

[19] Horozov TS, Aveyard R, Clint JH, Binks BP. Order-disorder transition in monolayers of modified monodisperse silica particles at the octane-water interface. Langmuir. 2003;19:2822-2829. DOI: 10.1021/la020858x

[20] Peng B, Smallenburg F, Imhof A, Dijkstra M, van Blaaderen A. Colloidal clusters by using emulsions and dumbbell-shaped particles: Experiments and simulations. Angew Chem Int Edit. 2013;52:6709-6712. DOI: 10.1002/anie.201301520

[21] Kim S-H, Lee SY, Yi G-R, Pine DJ, Yang S-M. Microwave-assisted self-organization of colloidal particles in confining aqueous droplets. J Am Chem Soc. 2006;128:1089710904. DOI: $10.1021 /$ ja063528y

[22] Yi G-R, Thorsen T, Manoharan VN, Hwang M-J, Jeon S-J, Pine DJ, Quake SR, Yang SM. Generation of uniform colloidal assemblies in soft microfluidic devices. Adv Mater. 2003;15:1300-1304. DOI: 10.1002/adma.200304890

[23] Zerrouki D, Rotenberg B, Abramson S, Baudry J, Goubault C, Leal-Calderon F, Pine DJ, Bibette M. Preparation of doublet, triangular, and tetrahedral colloidal clusters by controlled emulsification. Langmuir. 2006;22:57-62. DOI: 10.1021/la051765t

[24] Buzzaccaro S, Tripodi A, Rusconi R, Vigolo D, Piazza R. Kinetics of sedimentation in colloidal suspensions. J Phys Condens Mat. 2008;20:1-9. DOI: 10.1088/09538984/20/49/494219 
[25] Plüisch CS, Wittemann A. Shape-tailored polymer colloids on the road to become structural motifs for hierarchically organized materials. Macromol Rapid Commun. 2013;34:1798-1814. DOI: 10.1002/marc.201300693

[26] Wagner CS, Lu Y, Wittemann A. Preparation of submicrometer-sized clusters from polymer spheres using ultrasonication. Langmuir. 2008;24:12126-12128. DOI: 10.1021/la802580k

[27] Wagner CS, Fischer B, May M, Wittemann A. Templated assembly of polymer particles into mesoscopic clusters with well-defined configurations. Colloid Polym Sci. 2010;288:487-498. DOI: 10.1007/s00396-009-2169-y

[28] Landfester K. Recent developments in miniemulsions - formation and stability mechanisms. Macromol Symp. 2000;150:171-178. DOI: 10.1002/15213900(200002)150:1<171::AID-MASY171>3.0.CO;2-D

[29] van Blaaderen A. Chemistry: Colloidal molecules and beyond. Science. 2003;301:470471. DOI: $10.1126 /$ science. 1087140

[30] Frenkel D, Wales DJ. Colloidal self-assembly: Designed to yield. Nat Mater. 2011;10:410-411. DOI: 10.1038/nmat3037

[31] Meng G, Arkus N, Brenner MP, Manoharan VN. The free-energy landscape of clusters of attractive hard spheres. Science. 2010;327:560-563. DOI: 10.1126/science.1181263

[32] Schwarz I, Fortini A, Wagner CS, Wittemann A, Schmidt M. Monte Carlo computer simulations and electron microscopy of colloidal cluster formation via emulsion droplet evaporation. J Chem Phys. 2011;135:244501. DOI: 10.1063/1.3672106

[33] Binks BP. Particles as surfactants - similarities and differences. Curr Opin Colloid Interface. 2002;7:21-41. DOI: 10.1016/S1359-0294(02)00008-0

[34] Binks BP, Horozov TS, editors. Colloidal particles at liquid interfaces. Cambridge: Cambridge University Press; 2006. 501 p. ISBN: 978-0521-071314

[35] Pazos-Perez N, Wagner CS, Romo-Herrera JM, Liz-Marzán LM, García de Abajo FJ, Wittemann A, Fery A, Alvarez-Puebla RA. Organized plasmonic clusters with high coordination number and extraordinary enhancement in surface-enhanced raman scattering (sers). Angew Chem Int Edit. 2012;51:12688-12693. DOI: 10.1002/anie. 201207019

[36] Radziuk D, Möhwald H. Ultrasonically treated liquid interfaces for progress in cleaning and separation processes. Phys Chem Chem Phys. 2016;18:21-46. DOI: 10.1039/ c5cp05142h

[37] Tadros TF, editor. Emulsion science and technology: A general introduction. Weinheim: Wiley-VCH; 2009. 1-56 p. ISBN: 978-3-527-32525-2

[38] Gilbert RG. Emulsion polymerization: A mechanistic approach. London: Academic Press; 1995. ISBN: 978-0122830600 
[39] Maranas CD, Floudas CA. A global optimization approach for Lennard-Jones microclusters. J Chem Phys. 1992;97:7667-7678. DOI: 10.1063/1.463486

[40] Romo-Herrera JM, Alvarez-Puebla RA, Liz-Marzán LM. Controlled assembly of plasmonic colloidal nanoparticle clusters. Nanoscale. 2011;3:1304-1315. DOI: 10.1039/ c0nr00804d

[41] Rodriguez-Fernandez J, Perez-Juste J, García de Abajo FJ, Liz-Marzán LM. Seeded growth of submicron au colloids with quadrupole plasmon resonance modes. Langmuir. 2006;22:7007-7010. DOI: 10.1021/la060990n

[42] Wagner CS, Shehata S, Henzler K, Yuan J, Wittemann A. Towards nanoscale composite particles of dual complexity. J Colloid Interface Sci. 2011;355:115-123. DOI: 10.1016/ j.jcis.2010.12.017

[43] Wagner CS, Fortini A, Hofmann E, Lunkenbein T, Schmidt M, Wittemann A. Particle nanosomes with tailored silhouettes. Soft Matter. 2012;8:1928-1933. DOI: 10.1039/ C1SM07014B

[44] Fritsch A. Preparative density gradient centrifugations. Geneva: Beckman Instruments; 1975.

[45] Hoffmann M, Wagner CS, Harnau L, Wittemann A. 3D brownian diffusion of submicron-sized particle clusters. ACS Nano. 2009;3:3326-3334. DOI: 10.1021/nn900902b

[46] Myers D. Surfaces, interfaces, and colloids: Principles and applications. New York, USA: Wiley-VCH; 1999. 528 p. ISBN: 978-0-471-33060-8

[47] Degiorgio V, Piazza R. Rotational diffusion in concentrated colloidal dispersions of hard spheres. Phys Rev E. 1995;52:2707-2717. DOI: 10.1103/PhysRevE.52.2707

[48] Lettinga MP, Koenderink GH, Kuipers BWM, Bessels E, Philipse AP. Rotational dynamics of colloidal spheres probed with fluorescence recovery after photobleaching. J Chem Phys. 2004;120:4517-4529. DOI: 10.1063/1.1644799

[49] Quirantes A, Ben-Taleb A, Delgado AV. Determination of size/shape parameters of colloidal ellipsoids by photon correlation spectroscopy. Colloids Surf A. 1996;119:7380. DOI: 10.1016/S0927-7757(96)03728-4

[50] Martchenko I, Dietsch H, Moitzi C, Schurtenberger P. Hydrodynamic properties of magnetic nanoparticles with tunable shape anisotropy: Prediction and experimental verification. J Phys Chem B. 2011;115:14838-14845. DOI: 10.1021/jp2078264

[51] de Souza Lima MM, Wong JT, Paillet M, Borsali R, Pecora R. Translational and rotational dynamics of rodlike cellulose whiskers. Langmuir. 2003;19:24-29. DOI: 10.1021/ la020475z

[52] Kröger A, Deimede V, Belack J, Lieberwirth I, Fytas G, Wegner G. Equilibrium length and shape of rodlike polyelectrolyte micelles in dilute aqueous solutions. Macromolecules. 2007;40:105-115. DOI: 10.1021/ma061966j 
[53] Jabbari-Farouji S, Eiser E, Wegdam GH, Bonn D. Ageing dynamics of translational and rotational diffusion in a colloidal glass. J Phys Condens Matter. 2004;16:L471-L477. DOI: 10.1088/0953-8984/16/41/L07

[54] Plum MA, Steffen W, Fytas G, Knoll W, Menges B. Probing dynamics at interfaces: Resonance enhanced dynamic light scattering. Opt Express. 2009;17:10364-10371. DOI: 10.1364/OE.17.010364

[55] Heyes DM, Nuevo MJ, Morales JJ, Branka AC. Translational and rotational diffusion of model nanocolloidal dispersions studied by molecular dynamics simulations. J Phys Condens Matter. 1998;10:10159-10178. DOI: 10.1088/0953-8984/10/45/005

[56] Aragon SR, Pecora R. Theory of dynamic light scattering from large anisotropic particles. J Chem Phys. 1977;66:2506-2516. DOI: 10.1063/1.434246

[57] Hoffmann M, Lu Y, Schrinner M, Ballauff M, Harnau L. Dumbbell-shaped polyelectrolyte brushes studied by depolarized dynamic light scattering. J Phys Chem B. 2008;112:14843-14850. DOI: 10.1021/jp806765y

[58] Anthony SM, Kim M, Granick S. Translation-rotation decoupling of colloidal clusters of various symmetries. J Chem Phys. 2008;129:244701-1-6. DOI: 10.1063/1.3043443

[59] Berne BJ, Pecora R. Dynamic light scattering: With applications to chemistry, biology, and physics. Mineola: Dover Publications; 2000. 384 p. ISBN: 978-0-486-41155-2

[60] Oetama RJ, Walz JY. Simultaneous investigation of sedimentation and diffusion of a single colloidal particle near an interface. J Chem Phys. 2006;124:164713-1-8. DOI: $10.1063 / 1.2189853$

[61] Brogioli D, Salerno D, Cassina V, Sacanna S, Philipse AP, Croccolo F, Mantegazza F. Characterization of anisotropic nano-particles by using depolarized dynamic light scattering in the near field. Opt Express. 2009;17:1222-1233. DOI: 10.1364/OE.17.001222

[62] García de la Torre J, Del Rio Echenique G, Ortega A. Improved calculation of rotational diffusion and intrinsic viscosity of bead models for macromolecules and nanoparticles. J Phys Chem B. 2007;111:955-961. DOI: 10.1021/jp0647941

[63] Carrasco B, García de la Torre J. Hydrodynamic properties of rigid particles: Comparison of different modeling and computational procedures. Biophys J. 1999;76:30443057. DOI: 10.1016/S0006-3495(99)77457-6

[64] Liu K, Zhao NN, Kumacheva E. Self-assembly of inorganic nanorods. Chem Soc Rev. 2011;40:656-671. DOI: 10.1039/c0cs00133c

[65] Fan JA, Wu CH, Bao K, Bao JM, Bardhan R, Halas NJ, Manoharan VN, Nordlander P, Shvets G, Capasso F. Self-assembled plasmonic nanoparticle clusters. Science. 2010;328:1135-1138. DOI: 10.1126/science.1187949

[66] Kneipp K. Surface-enhanced raman scattering. Phys Today. 2007;60:40-46. DOI: 10.1063/1.2812122 
[67] Le Ru EC, Etchegoin PG. Single-molecule surface-enhanced raman spectroscopy. Annu Rev Phys Chem. 2012;63:65-87. DOI: 10.1146/annurev-physchem-032511-143757

[68] Abdullin TI, Bondar OV, Shtyrlin YG, Kahraman M, Culha M. Pluronic block copolymer-mediated interactions of organic compounds with noble metal nanoparticles for sers analysis. Langmuir. 2010;26:5153-5159. DOI: 10.1021/la9036309

[69] Alvarez-Puebla R, Contreras-Ciceres R, Pastoriza-Santos I, Perez-Juste J, Liz-Marzán LM. Au@pnipam colloids as molecular traps for surface-enhanced, spectroscopic, ultrasensitive analysis. Angew Chem Int Ed. 2009;48:138-143. DOI: 10.1002/anie.200804059

[70] Alvarez-Puebla RA, Liz-Marzán LM. Traps and cages for universal sers detection. Chem Soc Rev. 2012;41:43-51. DOI: 10.1039/C1CS15155J 